\title{
El comercio de Tailandia y su relación con México
}

Agustina Rodríguez Alegría*

$\mathrm{E}$ l comercio y la inversión extranjera directa (IED) constituyen una fuente de recursos importantes para la economía de Tailandia, motivo por el cual el gobierno de ese país implementó medidas para atraer recursos del exterior, orientándolos a sectores que coadyuven en el incremento del valor añadido en los productos de exportación.

Las políticas en materia de inversión y comercio han sido diseñadas con la finalidad de incrementar la apertura comercial del país, tomando como punto de referencia los acuerdos y recomendaciones del grupo de países que conforman el Área de Libre Comercio de la ASEAN (Asociación de Naciones del Sudeste Asiático), la Organización Mundial de Comercio (OMC) y el Fondo Monetario Internacional (FMI).

En 1990 el gobierno de Tailandia inicióla planeación de un cambio estratégico en la orientación de sus mercados: consideró de gran importancia dirigir una política de redistribución de las actividades económicas, descentralizando decisiones que hasta entonces eran facultad exclusiva del gobierno. Sin embargo, fue hasta 1994 cuando da inicio el cambio en el rumbo de la economía, al apoyarse en la planificación socioeconómica dividida en planes quinquenales -los cuales han sido utilizados desde 1960-y enfocarse en el ajuste estructural que abarca principalmente áreas estratégicas como: el proceso de diversificación en el sector agrícola, la conformación de un plan estratégico exportador y la creación de un marco legal de fomento a la competencia, para regular el comercio y la inversión.

* Investigadora del Departamento de Estudios del Pacífico de la Universidad de Guadalajara.

\section{La inversión}

En 1997 Tailandia anunció un plan maestro de privatización de algunas empresas como parte del plan estratégico de desregulación gubernamental y de liberalización comercial, con el objeto de corregir el desajuste económico que empezó a manifestarse a mediados de los noventa. En ese proceso se optó por la privatización de industrias clave en la economía: la petroquímica, las telecomunicaciones, el acero, la generación de energía y la industria automovilística integrada. Así, se alentó a la industria intensiva en capital y coadyuvó al proceso de transferencia de tecnología y a la creación de empleos, así como al ingreso de divisas.

La IED juega un papel importante en la economía tailandesa, incluso la desaceleración de la economía se atribuye a la disminución en la inversión proveniente el exterior. Durante 1995, 70 por ciento de las inversiones provinieron del exterior. La industria tailandesa ha mostrado recientemente un cambio en su composición, al pasar de ser intensiva en mano de obra a ser intensiva en capital, con la adquisición de un alto nivel tecnológico; aunque ello requirió de mayores gastos financieros para su funcionamiento.

De esta forma, y con base en los objetivos planteados en los planes quinquenales, se desarrolló la industria petroquímica, la cual ha requerido de la construcción de plantas de refinería y procesamiento químico. El apoyo que recibe este sector se sustenta en una menor dependencia de la importación de productos. En el ramo de las telecomunicaciones destacan las compañías telefónicas, cuya finalidad principal fue lograr una modernización tecnológica de la industria mediante la participación de capital privado y externo. En lo que respecta al sector automotriz, Tailandia se ha convertido en uno 
de los centros mundiales de ensamblaje de autos, ya que importantes firmas niponas y estadounidenses tienen centros de ensamblaje en ese país (por ejemplo, Toyota, Honda, Nissan son de origen Japonés; General Motors, de Estados Unidos). De esta manera, Tailandia se ha convertido en una plataforma exportadora de autos para los países de la región asiática.

\section{El comercio}

Hasta finales de los ochenta Tailandia basó sus exportaciones principalmente en los productos primarios, y aun en la actualidad la OMC le considera como parte del grupo de los quince países más importantes en la exportación de productos agrícolas en 1999.

Las nuevas medidas encaminadas a lograr la apertura económica tienen como finalidad el impulso a la industria manufacturera, lo cual garantiza un alto valor añadido en sus productos $\mathrm{y}$ una menor dependencia de las fluctuaciones inherentes a los precios de los productos agrícolas. Se ha puesto especial atención al desarrollo de las ramas industriales como la textil, el ensamblaje de autos, la de equipos eléctricos, la industria del acero y el procesamiento de alimentos. Recientemente, han cobrado importancia las exportaciones de equipo y componentes electrónicos, circuitos integrados, computadoras y sus componentes. El comercio de servicios también se ha visto impulsado, destacando el transporte, telégrafos, teléfonos y el turismo.

Las medidas anteriores han dado como resultado un cambio estructural en las exportaciones. Así, en 1990 los productos agrícolas representaban 33.7 por ciento de las exportaciones totales en Tailandia, que se redujo a 20.1 por ciento en 1999 .
El comercio total tailandés registró un incremento en el volumen del flujo comercial, al pasar de 77 millones de dólares en 1993 a 99 millones de dólares en 1999. No obstante su ritmo de crecimiento, el comercio se ha visto afectado, ya que en el período anterior a la aplicación de las políticas comerciales de ese país 1980-1993, mantuvo un crecimiento promedio anual de las exportaciones de 15.6 por ciento, que cae a 8.3 por ciento en el período 1994-1999. En lo que respecta a las importaciones, también se observa una considerable reducción, al pasar de 15 por ciento en el primer período a 8 por ciento en el segundo.

Tailandia ha alentado el comercio regional con los países miembros de la ASEAN, con los que conforman el Foro de Cooperación Económica de Asia Pacífico (APEC), y con algunos países de Europa. En consecuencia, el flujo comercial con esas regiones se ha incrementado: de 23.0 por ciento de sus exportaciones totales en 1990 , a 54.4 por ciento en 1998 y 58.4 en 1999. De igual forma, se observa incremento en las importaciones procedentes de esos grupos de países, ya que en 1990 constituían 33.38 de las importaciones totales y para 1999, el 50.3 por ciento.

Los principales socios comerciales de Tailandia son EU, Japón y Singapur, sumando los tres 45.7 por ciento respecto al total de sus exportaciones. Estados Unidos representa el primer lugar, con 19.4 por ciento; en segundo lugar está Japón, con 15.2 por ciento y el tercero lo ocupa Singapur, con 11.1 por ciento. En las importaciones figuran los tres países mencionados sumando un total de 44.5 por ciento del total. En este caso, Japón mantiene el primer lugar, con una participación de 25.7 por ciento; en segundo lugar, Estados Unidos, con 13.8 por ciento y Singapur solamente 5.0 por ciento respecto a las importaciones totales en 1997 (cuadro 1). 
Cuadro 1

Tailandia: socios comerciales, países seleccionados

(porcentajes)

\begin{tabular}{|c|c|c|c|c|c|c|c|}
\hline & 1991 & 1992 & 1993 & 1994 & 1995 & 1996 & 1997 \\
\hline Exportaciones ( mdd ) & $28,811.0$ & $32,472.0$ & $37,158.0$ & $45,583.0$ & $57,200.0$ & $55,743.0$ & $57,518.0$ \\
\hline Total & 100.0 & 100.0 & 100.0 & 100.0 & 100.0 & 100.0 & 100.0 \\
\hline Total regional & 65.8 & 68.1 & 68.8 & 71.4 & 68.6 & 70.4 & 71.0 \\
\hline Estados Unidos & 21.1 & 22.5 & 21.5 & 20.9 & 17.6 & 18.0 & 19.4 \\
\hline Japón & 17.8 & 17.5 & 17.0 & 17.0 & 16.6 & 16.8 & 15.2 \\
\hline Singapur & 8.1 & 8.7 & 12.0 & 13.5 & 13.8 & 12.1 & 11.1 \\
\hline Reino Unido & 3.6 & 3.6 & 3.2 & 3.0 & 2.8 & 3.3 & 3.5 \\
\hline Holanda & 4.3 & 4.3 & 3.1 & 2.8 & 3.1 & 3.2 & 3.2 \\
\hline Hong Kong & 4.7 & 4.6 & 5.3 & 5.2 & 5.1 & 5.8 & 5.9 \\
\hline China & 1.2 & 1.2 & 1.2 & 2.0 & 2.9 & 3.4 & 3.0 \\
\hline Malasia & 2.4 & 2.6 & 2.8 & 3.7 & 2.7 & 3.6 & 4.3 \\
\hline Taiwán & 1.6 & 1.9 & 2.0 & 2.2 & 2.4 & 2.5 & 2.7 \\
\hline Indonesia & 0.7 & 0.9 & 0.5 & 1.0 & 1.4 & 1.5 & 2.4 \\
\hline México & 0.3 & 0.3 & 0.2 & 0.2 & 0.1 & 0.1 & 0.2 \\
\hline Otros & 34.2 & 31.9 & 31.2 & 28.6 & 31.4 & 29.6 & 29.0 \\
\hline Importaciones (mdd) & $37,925.0$ & $40,686.0$ & $46,065.0$ & $54,394.0$ & $73,692.0$ & $73,336.0$ & $62,875.0$ \\
\hline Total & 100.0 & 100.0 & 100.0 & 100.0 & 100.0 & 100.0 & 100.0 \\
\hline Total regional & 64.3 & 66.2 & 64.7 & 66.2 & 63.5 & 63.2 & 63.6 \\
\hline Estados Unidos & 10.5 & 11.7 & 11.7 & 11.9 & 11.5 & 12.6 & 13.8 \\
\hline Japón & 29.1 & 29.3 & 30.3 & 30.2 & 29.3 & 27.9 & 25.7 \\
\hline Singapur & 7.9 & 7.3 & 6.4 & 6.3 & 5.6 & 5.5 & 5.0 \\
\hline Reino Unido & 2.2 & 2.3 & 2.3 & 2.1 & 2.0 & 2.2 & 2.1 \\
\hline Holanda & 0.9 & 1.0 & 0.9 & 0.9 & 0.9 & 0.8 & 1.0 \\
\hline Hong Kong & 2.0 & 1.2 & 1.2 & 1.3 & 1.0 & 1.2 & 1.3 \\
\hline China & 3.0 & 3.0 & 2.0 & 2.6 & 2.8 & 2.7 & 3.6 \\
\hline Malasia & 3.1 & 3.9 & 3.6 & 4.9 & 4.4 & 4.9 & 4.8 \\
\hline Taiwán & 4.7 & 5.5 & 5.1 & 5.1 & 4.6 & 4.0 & 4.6 \\
\hline Indonesia & 0.6 & 0.7 & 1.1 & 0.8 & 0.9 & 1.3 & 1.4 \\
\hline México & 0.2 & 0.1 & 0.1 & 0.1 & 0.2 & 0.2 & 0.3 \\
\hline Otros & 35.7 & 33.8 & 35.3 & 33.8 & 36.5 & 36.8 & 36.4 \\
\hline
\end{tabular}

Fuente: Monetary Fund, Direction of Trade Statistics, 1997.

\section{Política arancelaria tailandesa}

En la década de los noventa se avanzó mucho en la desgravación arancelaria, abarcando alrededor de 4000 artículos. El arancel promedio aplicado pasó de 30 por ciento en 1994 a 17 por ciento en 1997. Cabe mencionar que se exceptúan algunos productos, principalmente aquellos que son considerados no aptos para competir actualmente con las mercancías extranjeras, entre las cuales podemos mencionar los automóviles, cuyo arancel promedio se 
mantiene en 38 por ciento, aunque en algunos casos llegan a mantener un porcentaje de 80 . Entre los productos que han mostrado mayor reducción en los aranceles destacan el equipo médico y fertilizantes (que han quedado libres); las materias primas, los componentes electrónicos y los vehículos de transporte internacional se redujeron a 1 por ciento; los bienes de capital, maquinaria y computadoras, a 5 por ciento; los bienes intermedios, a 10 por ciento. Los bienes que presentan una mayor protección que los anteriores son: refrigeración y aire acondicionado, carne, lácteos, frutas, vegetales, granos, arroz, azúcar, algunas bebidas, tabaco, prendas de vestir y automóviles, los cuales mantienen un promedio arancelario de 30 por ciento, y algunos casos específicos alcanzan hasta 60 y 80 por ciento. En cambio, los aranceles sobre los productos intermedios son reducidos a cero cuando las importaciones de estos bienes requieren un proceso industrial doméstico.

\section{La inversión de México y Tailandia}

De acuerdo con datos proporcionados por la Secretaría de Economía, la inversión extranjera que llegó a México en el período 1994-2000 ascendió a 65697 millones de dólares. De este total, 61 por ciento provienen de Estados Unidos, 22 por ciento de la Unión Europea y 5.8 por ciento de países de la Cuenca del Pacífico. En esa última región, entre los que figuran por su mayor participación en la IED mexicana se encuentran Japón, con 76 por ciento respecto al total de inversión, Corea del Sur con 12 por ciento, Singapur con 6.1 por ciento, Taiwán con 1.7 por ciento, China 1 por ciento y Tailandia con 0.4 por ciento.

Hasta septiembre de 2000, se registraron siete empresas con participación de capital proveniente de Tailandia. Entre enero de 1994 y septiembre de 2000, las empresas con capital tailandés concentraron inversiones en México por 17.1 millones de dólares. De esta forma, ocuparon la octava posición entre los países de la Cuenca del Pacífico que, en ese lapso, materializaron inversiones en México. El 97 por ciento de la IED de origen tailandés se destinó al sector manufacturero y 3 por ciento al sector agropecuario.

De las siete empresas tailandesas instaladas en México, dos están dedicadas al sector agropecuario, dos a la industria manufacturera (una elabora productos de plástico y la otra fabrica y ensambla maquinaria y equipo para usos generales); en el sector comercio se concentran dos empresas (una se dedica al comercio de productos no alimenticios y la otra en alimentos bebidas y tabaco); por último, tenemos una empresa dedicada a servicios financieros.

\section{El comercio de México y Tailandia}

México y Tailandia tienen en común haber implementado políticas comerciales encaminadas hacia una apertura significativa que los hará más competitivos y con una visión amplia del comercio global.

El comercio entre México y Tailandia no es significativo respecto al volumen y valor característica que se comparte con la gran mayoría de los países de Asia Pacífico.

Como ya se ha mencionado en otras colaboraciones publicadas en esta revista, cuando hemos abordado la relación comercial con algunos países de esa zona, el principal socio comercial de México es Japón, y lo ha sido por mucho tiempo. Cabe resaltar que desde mediados de los ochenta México ha mantenido un déficit comercial con los países de la Cuenca del Pacífico, mostrando un incremento cada vez mayor, y Tailandia no es la excepción al respecto.

México y Tailandia no figuran mutuamente como socios comerciales importantes; sin embargo, podemos observar un comercio con una tendencia ascendente, aunque el mayor incremento ha sido en las importaciones mexicanas provenientes de ese país, mostrando así un déficit creciente para México. En 1991 el volumen de exportaciones fue de apenas 4 millones de dólares, alcanzando su nivel más alto en la historia en 1997 cuando ascendió a 123 millones de dólares; no 


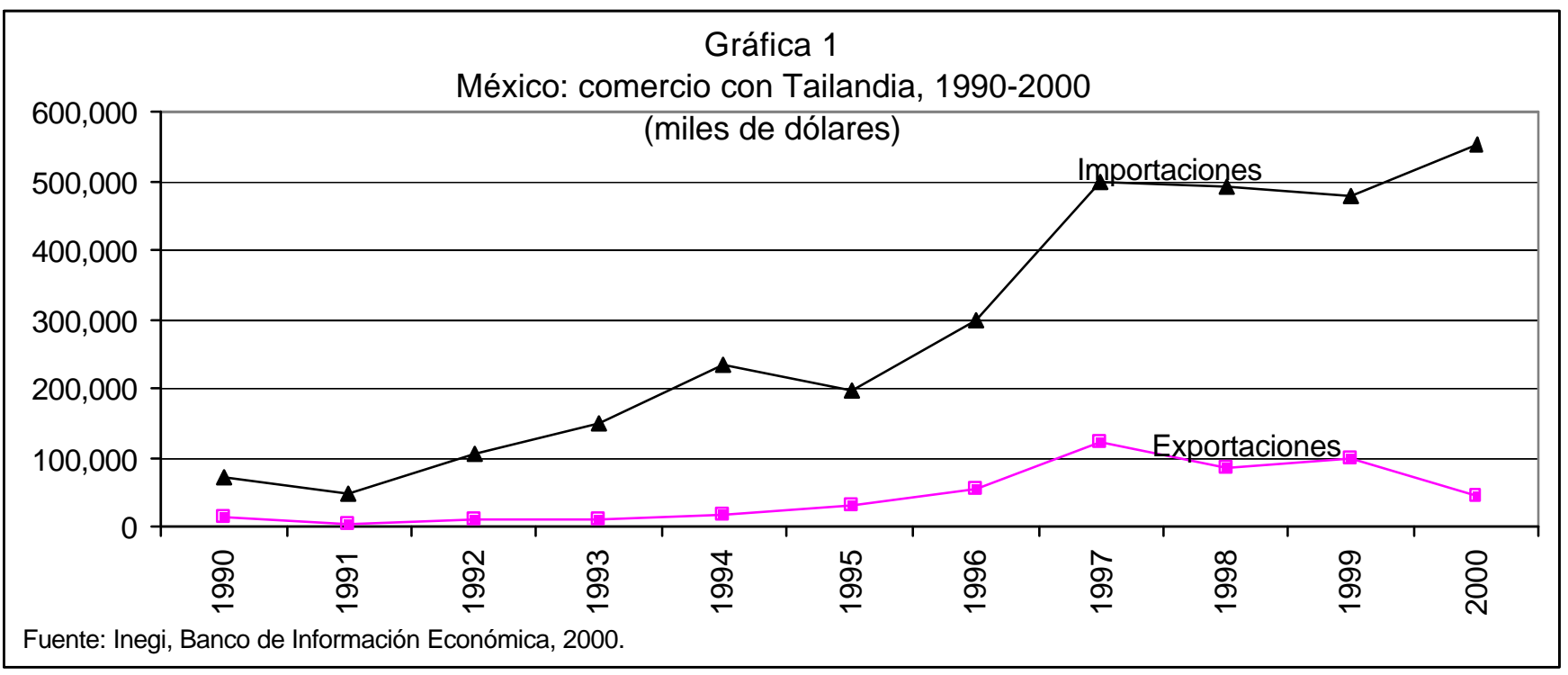

obstante, en 1999 éstas disminuyeron a 98 millones de dólares (ver gráfica).

La composición de las exportaciones se encuentra principalmente en partes y accesorios para máquinas de oficina. Este rubro constituye 75 por ciento respecto a las exportaciones mexicanas a Tailandia, mostrando un crecimiento de 8.2 por ciento en 1999 , respecto al año anterior (cuadro 2). En cuanto a las importaciones mexicanas provenientes de Tailandia han mantenido una tendencia creciente, al pasar de 43 millones de dólares en 1991 a 379 millones en 1999. La composición de las importaciones se concentra en partes para motor, las cuales representan 32 por ciento, mientras que las computadoras participan con 11.1 por ciento, constituyendo estos dos productos 43 por ciento del total (cuadro 2 ).

\section{Comentarios finales}

Tailandia, al igual que muchos países en el mundo, se jacta de ser un país con una apertura al comercio cada vez más creciente, como una forma de remediar los obstáculos que enfrenta en su economía y lograr un avance integral. No obstante el impulso que se le ha dado al comercio y la gran apertura a la inversión, no se ha logrado una total diversificación al destino y origen del comercio, ya que sus principales socios siguen siendo Estados Unidos, Japón y Singapur.

La pregunta sería por qué a pesar de las políticas implementadas respecto al comercio y la inversión no disminuye la dependencia entre unos cuantos países socios. Al igual que para México, la dependencia es mayor con EU. Cabe esperar que las condiciones mejoren con el tiempo al consolidarse los acuerdos o tratados comerciales con otros países. La reforma a las políticas comerciales ha cobrado vital importancia en varios países del mundo, como una tendencia globalizadora que rige la transformación de las economías, donde se busca reorientar los mercados para impulsar su economía y no quedarse fuera de esa corriente liberalizadora.

Según la historia reciente en materia comercial, México y Tailandia son mercados potenciales el uno del otro, por tal motivo se debe poner atención en las ventajas que se ofrecen mutuamente, así como encontrar la manera de aprovechar esas políticas de apertura entre ambos.

Por otro lado, el país con mayor importancia en aspectos comerciales y de inversión en México es Japón, mientras que Tailandia es uno de los países de menor comercio e inversión localizados en México. No obstante 
los posibles acuerdos comerciales que han anunciado el gobierno de México con Japón y Singapur, podría ser motivo de mayor acercamiento a esos países de Asia Pacífico y en un futuro no muy lejano conquistar esos mercados asiáticos.

\section{Fuentes}

Foreign Trade Barriers in Thailand, en: http://www.usisaustralia.gov/hyper/WF980401/epf313.htm. Foreigns Trade and Balance of Payments, en http:// www.mahidol.ac.th/Thailand/economy/foreign.html. STAT-USA, Country Commercial Guide for Thailand. Secretaría de Economía, Subsecretaría de Negociaciones Comerciales Internacionales, Dirección General de inversión Extranjera, Inversión de Tailandia en México, septiembre de 2000. Escalona, Agüero Alejandro, Tailandia, Asia Pacífico, Centro de Estudios de Asia y África, México, El Colegio de México, 1997. International Monetary Fund, Direction of Trade Statistics Yearbook, 1998. $\mathrm{T!}:$

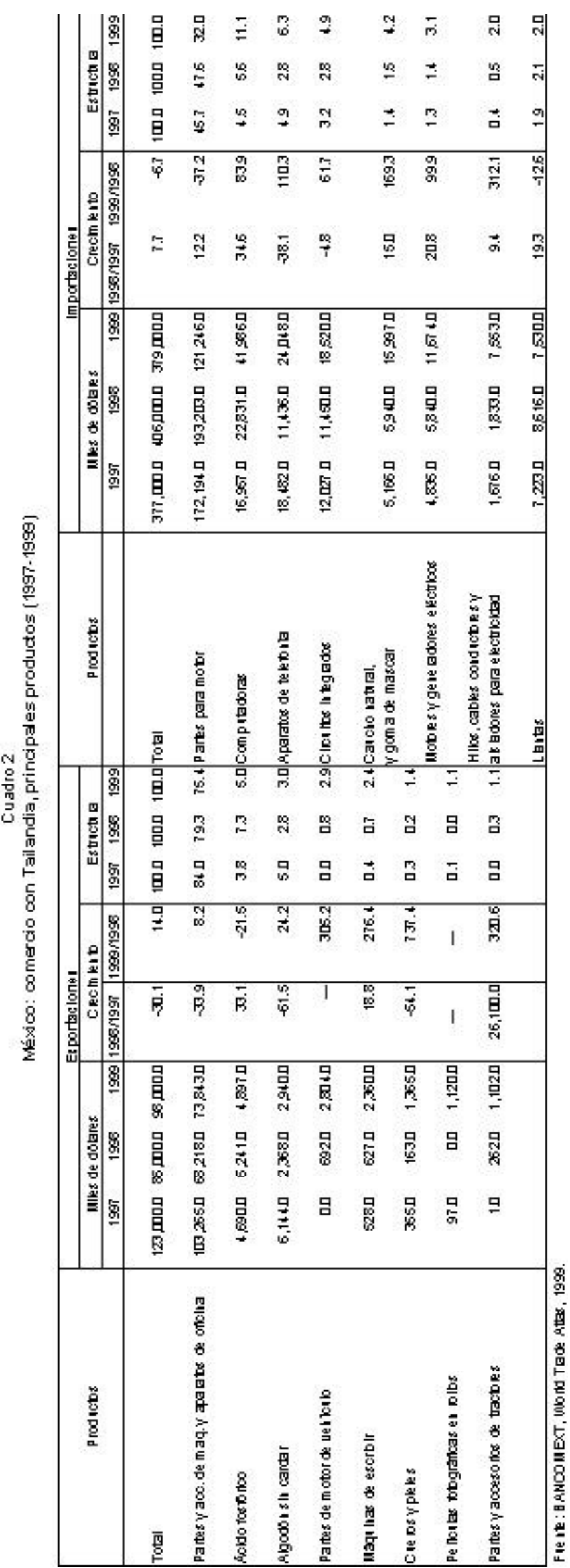

